\title{
Differential Cytokine Levels in Adults Induced by a Novel Candidate TB Boost Vaccine, MVA85A-According to Previous BCG Vaccination Status
}

Patrick K. Owiafe ${ }^{1}$, Philip C. Hill ${ }^{1,2}$, Hannah B. Ibanga ${ }^{1,3}$, Roger H. Brookes ${ }^{1,4}$, Helen McShane ${ }^{5}$, Jayne S. Sutherland ${ }^{1 *}$ and Martin O.C. Ota $^{1}$

${ }^{1}$ Vaccinology Theme, Medical Research Council (MRC) Unit, Fajara, The Gambia

${ }^{2}$ Centre for International Health, University of Otago, New Zealand

${ }^{3}$ Paediatrics, Queen's Hospital, Belvedere Road, Burton on Trent, UK

${ }^{4}$ Sanofi Pasteur, Connaught Campus, West Toronto, Ontario, Canada

${ }^{5}$ The Jenner Institute, University of Oxford, Roosevelt Drive, Oxford, UK

\begin{abstract}
MVA85A is a candidate boost vaccine for TB. A previously reported study showed no differences in response to MVA85A in 10 BCG vaccinated (BCG+) and 11 BCG naive (BCG-) adult Gambians. Here we evaluated the effect of pre-existing plasma cytokines in both groups before and after MVA85A vaccination on the vaccine-induced immune response. Pre-vaccination levels of IL-8 were higher in BCG+ subjects, whilst MCP-1 levels were lower compared to BCG-. Following MVA85A vaccination, concentrations of IL-8, IL-18, IL-12(p70) and MCP-1 were differentially induced between BCG+ and BCG- groups. The implications of these findings depend on their role in TB pathogenesis.
\end{abstract}

Keywords: Tuberculosis; Cytokines; MVA85A; BCG

\section{Introduction}

Tuberculosis (TB) remains a major public health concern worldwide, with more than 2 million deaths every year, particularly in sub-Saharan Africa and South East Asia [1]. This disease burden demonstrates that although BCG protects against severe forms of TB in children its effect is incomplete [2]. However, due to some other non-specific beneficial effects such as the prevention of childhood mortality, allergies and promotion of immune responses to other Expanded Programme of immunisation (EPI) vaccines [3-5], one current intervention strategy is to develop a new vaccination regimen that incorporates BCG, instead of replacing it.

Modified Vaccine virus Ankara expressing antigen 85A from Mycobacterium tuberculosis (M.tb) (MVA85A) is the most clinically advanced candidate vaccine developed to boost the effects of BCG. MVA85A was safe well tolerated and immunogenic when administered to adult volunteers in the UK, The Gambia and South Africa [6-8]. Interestingly, in The Gambian study, the immunogenicity of MVA85A in BCG vaccinated (BCG+) and BCG naive (BCG-) adults was comparable, in contrast to studies in the UK where immunogenicity was higher in BCG vaccinated subjects [6]. Furthermore in South Africa, the immunogenicity of MVA85A in BCG vaccinated adults was also comparable to that seen in BCG naive adults [8]. These findings suggest that MVA85A may boost cumulative mycobacterial exposure which is comprised of exposure to BCG, non-tuberculous mycobacteria and $M . t b$, and that the contribution of BCG to this cumulative mycobacterial exposure is less relevant in adults in TB endemic countries. To gain a better understanding of the relevance of baseline anti-mycobacterial immunity, we examined the cytokine and chemokine profiles in plasma obtained before and after MVA85A vaccination according to the presence or absence of a BCG scar in these healthy Gambian adults that have been previously reported [7].

\section{Methods}

\section{Study population}

This study was conducted between 2003 and 2005 and the setting and inclusion/exclusion criteria have been previously described [7].
10 subjects with a visible BCG scar (an indicator of BCG vaccination status for BCG [7]) and another 11 without evidence of a scar of BCG vaccination were included in the study. The groups did not differ significantly with respect to age, height, weight and Mantoux skin test and all subjects were HIV seronegative. None of the subjects had a previous history of asthma, diabetes or TB and their chest X-rays were normal. After written informed consent, blood samples were taken before and after MVA85A vaccination into heparinised tubes and processed to obtain peripheral blood mononuclear cells and plasma. Plasma samples were aliquoted and stored at -200C until required for the measurement of cytokines in this study. Clinical trial number NCT00423839 (clinicaltrials.gov).

\section{Multiplex bead array assay}

Plasma samples before and one week after vaccination were analysed using a Bio-Rad 17-plex kit according to the manufacturer's instructions. $50 \mu \mathrm{l}$ of bead suspension was added to each well and washed twice. $50 \mu$ of samples and standards were added and the plate incubated for 1 hour at $300 \mathrm{rpm}$. The plate was washed 3 times then $25 \mu \mathrm{l}$ of detection antibody added and the plate incubated for $30 \mathrm{~min}$. After washing, $50 \mu \mathrm{l}$ of $1 \mathrm{X}$ Streptavidin-PE (Phycoerythrin) was added to each well and incubated for $10 \mathrm{~min}$. The plate was again washed and resuspended in $125 \mu$ of assay buffer for reading using Bio-plex manager software (version 4.0) and a low photomultiplier tube setting.

\section{Statistical analysis}

Cytokine levels were log transformed and analysed within groups

*Corresponding author: Dr. J. Sutherland, Vaccinology Theme, MRC Unit, The Gambia, E-mail: jsutherland@mrc.gm

Received November 19, 2012; Accepted November 28, 2012; Published November 30, 2012

Citation: Owiafe PK, Hill PC, Ibanga HB, Brookes RH, McShane H, et al. (2012) Differential Cytokine Levels in Adults Induced by a Novel Candidate TB Boost Vaccine, MVA85A-According to Previous BCG Vaccination Status. J Vaccines Vaccin 3:158. doi:10.4172/2157-7560.1000158

Copyright: @ 2012 Owiafe PK, et al. This is an open-access article distributed under the terms of the Creative Commons Attribution License, which permits unrestricted use, distribution, and reproduction in any medium, provided the original author and source are credited. 
pre- and post-vaccination using a paired Wilcoxon signed rank test. Levels were compared between BCG scar positive and scar negative subjects using a Mann-Whitney U-test and corrected for multiple comparisons.

\section{Results}

\section{Plasma cytokine concentration for BCG scar negative subjects following MVA85A vaccination}

The majority of cytokines measured were either low (IFN- $\gamma$, TNF- $\alpha$, IL-7, IL-12, IL-4, IL-5, IL-10, IL- 13, IL-17, G-CSF) or undetectable before and after vaccination with MVA85A (IL-1 $\beta$, IL-2, IL-6). Nevertheless, we saw detectable levels of IL-18 (median $[\mathrm{IQR}]=51[27-97] \mathrm{pg} / \mathrm{mL}), \mathrm{MCP}-1(66[51-76] \mathrm{pg} / \mathrm{ml})$ and MIP- $1 \beta$ $(76[65-101] \mathrm{pg} / \mathrm{ml})$ prior to vaccination (Table 1, Figures $1 \mathrm{~A}-\mathrm{C}$ ). Following vaccination of the scar-negative subjects no change in IL-18 was seen but MCP- 1 and MIP- $1 \beta$ concentrations were both significantly lower (52[41-65]; $\mathrm{p}=0.0098$ and $69[62-102] \mathrm{pg} / \mathrm{ml}$; $\mathrm{p}=0.0244$ respectively).

\section{Plasma cytokine concentration for BCG scar positive subjects following MVA85A vaccination}

As seen with the BCG scar negative subjects, the majority of cytokines measured before vaccination were either low (IFN- $\gamma$, TNF- $\alpha$, IL-7, IL-12, IL-4, IL-5, IL-10, IL-13, IL-17, G-CSF) or undetectable (IL$1 \beta$, IL-2, IL-6). Plasma concentration of most cytokines and chemokines did not change after vaccination except for IL- 8 and MIP- $1 \beta$ which both increased significantly after vaccination (median $[\mathrm{IQR}]=77[57-87]$ pre- and 112 [67-256] $\mathrm{pg} / \mathrm{ml}$ post-vaccination for MIP-1 $\beta$ and $25[17-$ 37] pre- and 43[31-199] pg/ml post-vaccination for IL-8 (Table 1 and Figures $1 \mathrm{C}$ and 1D). There was also a small but observable decrease in IFN- $\gamma$ concentrations post-vaccination $(4.3[0-18] \mathrm{pg} / \mathrm{ml}$ pre- and 2.6[0-3.5] pg/ml post-vaccination; $\mathrm{p}=0.0422$; Table 1 and Figure 1E).
A.

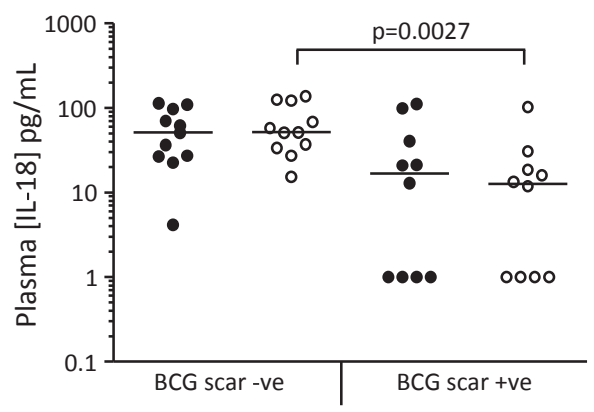

C.

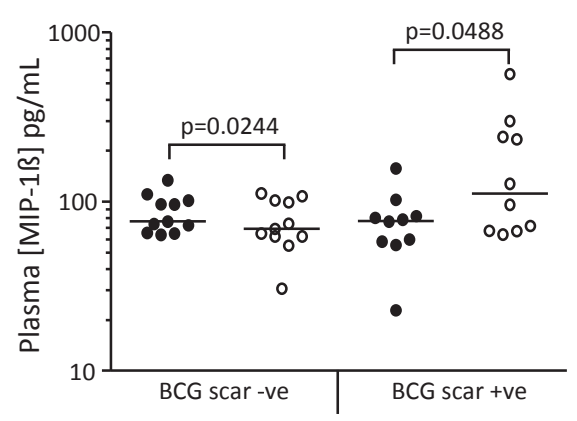

E.

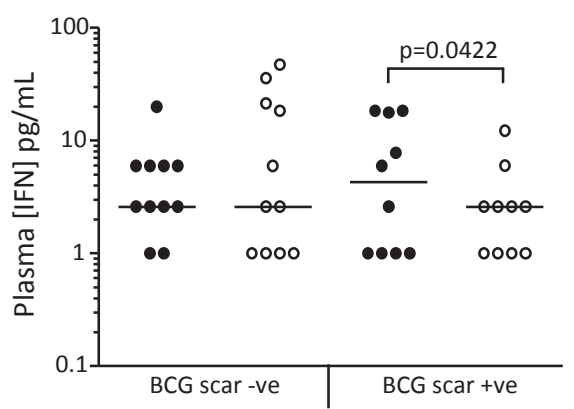

B. $p=0.0098$

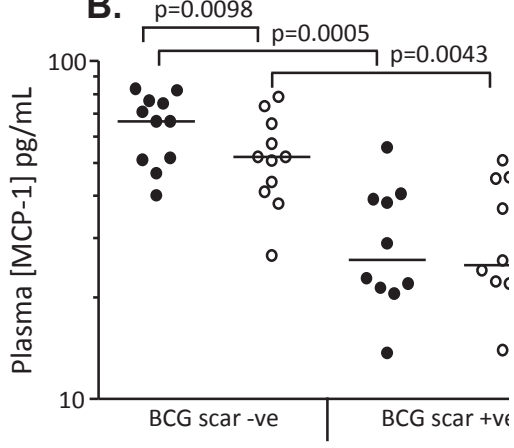

D.

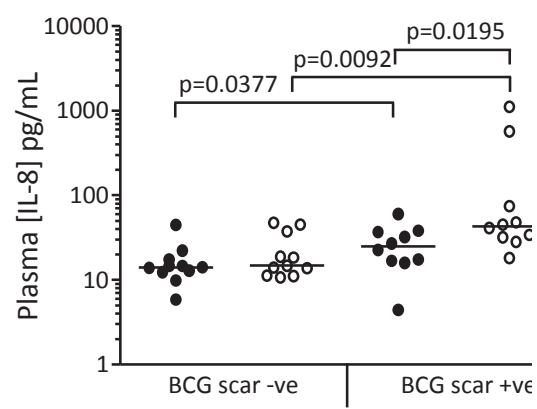

F.

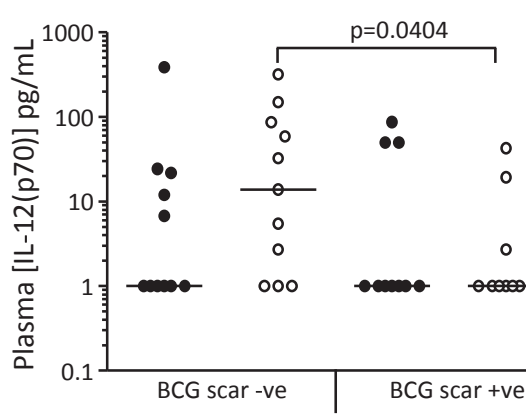

Figure 1: Cytokine and chemokine concentrations of BCG scar negative and scar positive healthy adult subjects pre and post vaccination. Pre-vaccination (filled) and post-vaccination (open).

Cytokine levels were analysed within groups using a paired Wilcoxon signed rank test. Levels were compared between BCG scar positive and scar negative subjects using a Mann-Whitney U-test. Pvalues are indicated. Line = median. 


\begin{tabular}{|c|c|c|c|c|}
\hline \multicolumn{3}{|c|}{ BCG scar negative } & \multicolumn{2}{|c|}{ BCG scar positive } \\
\hline Cytokine & Pre & Post & Pre & Post \\
\hline IFN-y & $2.6[2.6-5.9]$ & $2.6[0-21]$ & $4.3[0-18]$ & $2.6[0-3.5]$ \\
\hline TNF- $\alpha$ & $0[0-0]$ & $0[0-0]$ & $0[0-1]$ & $0[0-4.3]$ \\
\hline IL-7 & $8.1[2.4-22]$ & $3.7[1.4-7.3]$ & $2.3[0-45]$ & $6.3[3.9-43]$ \\
\hline IL-12(p70) & $0[0-22]$ & $14[0-87]$ & $0[0-50]$ & $59[0-106]$ \\
\hline IL-18 & $51[27-97]$ & $51[34-123]$ & $17[0-55]$ & $13[0-22]$ \\
\hline IL-4 & $0[0-3]$ & $0[0-0]$ & $0[0-0]$ & $0[0-0]$ \\
\hline IL-5 & $1.4[0.6-2.0]$ & $1.3[0.4-1.9]$ & $0.8[0-1.2]$ & $0.5[0.1-1.1]$ \\
\hline IL-13 & $0[0-0]$ & $0[0-0]$ & $0.7[0-12]$ & $0.4[0-11]$ \\
\hline IL-17 & $0[0-0]$ & $0[0-12]$ & $59[0-106]$ & $29[0-89]$ \\
\hline IL-10 & $3.7[1.4-7.3]$ & $4.4[1.3-5.3]$ & $4.1[2.7-9.6]$ & $4.2[2.8-8.6]$ \\
\hline MCP-1 & $66[51-76]$ & $52[41-65]$ & $26[21-39]$ & $25[22-45]$ \\
\hline MIP-1 $\beta$ & $76[65-101]$ & $69[62-102]$ & $77[57-87]$ & $112[67-256]$ \\
\hline IL-8 & $14[12-17]$ & $15[11-38]$ & $25[17-37]$ & $43[31-199]$ \\
\hline G-CSF & $0[0-17]$ & $0[0-54]$ & $0[0-19]$ & $0[0-9.5]$ \\
\hline IL-1 $\beta$ & $\mathrm{u} / \mathrm{d}$ & & & \\
\hline IL-2 & $u / d$ & & & \\
\hline IL-6 & $u / d$ & & & \\
\hline
\end{tabular}

$\mathrm{u} / \mathrm{d}=$ undetectable. Data expressed as median [interquartile range]

Table 1: Median cytokine responses for BCG scar-negative subjects before and after MVA85A vaccination

\section{Comparison of pre- and post-vaccination concentrations between BCG scar positive and negative subjects}

We next compared the cytokine concentrations for BCG scarnegatives and scar-positive subjects. While most cytokines were similar between the groups, IL-8 was significantly higher in scarpositive subjects compared to scar-negative subjects both before and after vaccination with MVA85A ( $\mathrm{p}=0.0377$ and $\mathrm{p}=0.0092$ respectively; Figure 1D). Conversely, MCP-1 was significantly lower in scar-positive compared to scar-negative subjects both before and after vaccination $(\mathrm{p}=0.0005$ and $\mathrm{p}=0.0043$ respectively; Figure $1 \mathrm{~B}$ ). Furthermore, IL-18 and IL12(p70) were both lower in scar-positive compared to scar-negative subjects after vaccination $(\mathrm{p}=0.0027$ and $\mathrm{p}=0.0404$ respectively; Figures $1 \mathrm{~A}$ and $1 \mathrm{~F})$.

\section{Discussion}

We assessed the effects of MVA85A vaccination on plasma cytokine levels depending on prior BCG status. In subjects with a BCG scar, MVA85A vaccination resulted in increases in IL- 8 and MIP- $1 \beta$ but a decrease in IFN- $\gamma$. In contrast, a decrease in both MCP- 1 and MIP-1 $\beta$ levels were seen following MVA85A vaccination of BCG scar-negative subjects. Interestingly, the concentration of MCP-1, IL-12(p70) and IL18 were all higher at post-vaccination in the BCG scar negative subjects compared to the BCG scar positive subjects whilst the reverse was the case for IL-8. These findings suggest that MVA85A has differential effects on the immune system depending on prior BCG vaccination status.

We have previously reported that vaccination with MVA85A induces comparable immune responses to Antigen 85A and PPD regardless of BCG status in Gambian adult subjects [7]. In contrast BCG scar positive subjects from the United Kingdom mounted a higher response to BCG naive subjects suggesting the threshold of responses is affected by environmental mycobacteria [6].

Cytokines and chemokines play a major role in the immune response to many infections. IL-8 is produced by macrophages and is one of the major mediators of the inflammatory response [9]. IL-8 was consistently higher in subjects with a BCG scar both before and after MVA85A vaccination. This reflects a boost effect of MVA85A on IL-8 suggesting that MVA is a proinflammatory agent. MCP-1 is required to attract monocytes and dendritic cells to sites of tissue inflammation and MIP- $1 \beta$ acts to induce activation of the macrophages $[10,11]$. Both these factors were lower after vaccination in BCG scar-positive subjects. Post-vaccination levels of IL-18 and IL- 12(p70) were also significantly lower in subjects who had received prior BCG vaccination. These results clearly show that in a TB endemic country setting, prior BCG vaccination modifies the response to MVA85A due to the pre-existing cytokine milieu. However, within subject groups, the scar negative group showed a decrease in MCP- 1 and MIP- $1 \beta$ with no other changes observed whereas the scar-positive group showed increases in MIP- $1 \beta$ and IL-8.

In conclusion, immunization with MVA85A induces differential changes in cytokines and chemokines which are dependent on prior BCG vaccination status. However, in the absence of defined immunological correlates of protection, the relevance of these findings for efficacy will only become clear when the results of ongoing trials are known.

\section{Acknowledgements}

We acknowledge the contributions of Adama Bojang and Abdulrahman Hammond and the TB Immunology lab staff. We also acknowledge the support of the Management of MRC Laboratories especially the former head of Bacterial Diseases Programme, Prof. Richard Adegbola. Above all we thank the study participants and their families.

\section{Funding}

The study was funded by the Medical Research Council (UK) and the European Commission (AFTBVAC-ICA4-CT-2002-10052). Neither funder had any role in the design of the study or the preparation of the manuscript.

\section{References}

1. WHO Report Global Tuberculosis control 2011.

2. Fine PE (1995) Variation in protection by BCG: implications of and for heterologous immunity. Lancet 346: 1339-1345.

3. Black GF, Weir RE, Floyd S, Bliss L, Warndorff DK, et al. (2002) BCG-induced increase in interferon-gamma response to mycobacterial antigens and efficacy of BCG vaccination in Malawi and the UK: two randomised controlled studies. Lancet 359: 1393-4101.

4. Ota MO, Vekemans J, Schlegel-Haueter SE, Fielding K, Sanneh M, et al. (2002) Influence of Mycobacterium bovis bacillus Calmette-Guerin on antibody and cytokine responses to human neonatal vaccination. J Immunol 168: 919-925.

5. Aaby P, Shaheen SO, Heyes CB, Goudiaby A, Hall AJ, et al. (2000) Early BCG vaccination and reduction in atopy in Guinea-Bissau. Clin Exp Allergy 30: 64450 .

6. McShane H, Pathan AA, Sander CR, Keating SM, Gilbert SC, et al. (2004) Recombinant modified vaccinia virus Ankara expressing antigen 85A boosts BCG-primed and naturally acquired antimycobacterial immunity in humans. Nat Med 10: 1240-1244.

7. Brookes RH, Hill PC, Owiafe PK, Ibanga BH, Jeffries JD, et al. (2008) Safety and immunogenicity of the candidate tuberculosis vaccine MVA85A in West Africa. PLoS One 3:e2921.

8. Scriba TJ, Tameris M, Mansoor N, Smit E, van der Merwe L, et al. (2010) Modified vaccinia Ankara-expressing Ag85A, a novel tuberculosis vaccine, is safe in adolescents and children, and induces polyfunctional CD4+ T cells. Eur J Immunol 40: 279-290.

9. Standiford TJ, Kunkel SL, Basha MA, Chensue SW, Lynch JP 3rd, et al. (1990) Interleukin-8 gene expression by a pulmonary epithelial cell line. A model for cytokine networks in the lung. J Clin Invest 86: 1945-1953.

10. Penido C, Vieira-de-Abreu A, Bozza MT, Castro-Faria-Neto HC, Bozza PT (2003) Role of monocyte chemotactic protein-1/CC chemokine ligand 2 on gamma delta $\mathrm{T}$ lymphocyte trafficking during inflammation induced by 
Citation: Owiafe PK, Hill PC, Ibanga HB, Brookes RH, McShane H, et al. (2012) Differential Cytokine Levels in Adults Induced by a Novel Candidate TB Boost Vaccine, MVA85A-According to Previous BCG Vaccination Status. J Vaccines Vaccin 3:158. doi:10.4172/2157-7560.1000158

Page 4 of 4

lipopolysaccharide or Mycobacterium bovis bacille Calmette-Guerin. J Immunol 171: $6788-6794$
11. Maurer M, Von Stebut E (2004) Macrophage inflammatory protein-1. Int $J$ Biochem Cell Biol 36: 1882-1886. 